\title{
MODELADO DE LA VELOCIDAD DE CORROSIÓN ATMOSFÉRICA DE ACERO DE BAJA ALEACIÓN EN COSTA RICA
}

\author{
Luis Garita Arce \\ Javier Rodríguez Yañez. \\ Javier Robles Cordero
}

\begin{abstract}
Resumen
A partir de datos de velocidad de corrosión atmosférica y variables ambientales en diferentes sitios de Costa Rica, se realizan evaluaciones matemáticas de los modelos usuales utilizados para la estimación de la velocidad de corrosión (Vcorr). Estos datos de contaminantes y parámetros atmosféricos en conjunto con la velocidad de corrosión obtenida gravimétricamente, son modelados por medio de la aplicación "optimtool” de Matlab , obteniendo resultados mixtos entre los modelos utilizados. Como principal conclusión se tiene el desarrollo de la Ecuación 3, la cual presenta un modelo de mejor ajuste para cada uno de los sitios, obteniéndo valores adecuados de los parámetros de control (RSS, F y $\mathrm{R}^{2}$ ), pero no así de la aplicación de las mismas en otros sitios.
\end{abstract}

Palabras clave: Corrosión atmosférica, modelado, velocidad de corrosión, acero de baja aleación

\begin{abstract}
From corrosion atmospheric rate data and its relationship with environmental variables of different Costa Rica locations, mathematical evaluations of typical models for corrosion rate estimation, were performed. Contamination data and atmospheric parameters along with gravimetric corrosion rate are modeled through Matlab's application "optimtool", yielding mixed results for the models. As main result, the development of equation 3, delivers a model of best adjustment for each site, which generates aceptable values for control parameters (RSS, F y $\mathrm{R}^{2}$ ), but not for the application of same model for other sites.
\end{abstract}

Key words: Atmospheric corrosion, modeling, corrosion rate, low alloy steel

Recibido: 22 de mayo de 2014 • Aprobado: 14 de julio de 2014

\section{INTRODUCCIÓN}

La corrosión atmosférica representa una de las principales causas de pérdida de materiales metálicos.

En Costa Rica se han realizado pocos estudios, generalmente asociados a evaluaciones financiadas por empresas o fondos internacionales, como fue el caso del Mapa Iberoamericano de Corrosión Atmosférica (MICAT) o el proyecto TROPICORR, pero todos de poca extensión en el tiempo o en pocos sitios (Morcillo, Almeida, Rosales, Uruchurtu, \& Morrocos, 1998) (Coto \& Saborio, 2006).

El Instituto Costarricense de Electricidad (ICE) realizó un estudio para evaluar la corrosión atmosférica en varias ubicaciones, considerando distintas regiones climáticas de interés, bajo los criterios de las normas ISO 9223 a ISO 9226 y otras normas asociadas (Coto \& Saborio, 2006) (ASTM, 1976). 
En base a esto se desarrolla según los diferentes criterios de modelados simples, la obtención de ecuaciones de aproximación adecuada a las características de Costa Rica.

\section{MATERIALES Y MÉTODOS}

\subsection{Obtención de datos}

La evaluación de la velocidad de corrosión atmosférica fue en acero de baja aleación conforme con las especificaciones de la norma ASTM A36, exponiendo las placas por 2 años para obtener la velocidad de corrosión gravimétrica (Davies, 1998). Además se realizó el monitoreo de los contaminantes, dióxido de azufre y cloruros de acuerdo a lo establecido en las normas ISO 9225 , igualmente se registraron por medio de estaciones meteorológicas automáticas del ICE los parámetros ambientales tales como: temperatura, humedad relativa y precipitación. (ISO, 1992)

Las estaciones utilizadas para la aplicación de los modelos son las indicadas en la Tabla 1. Los datos obtenidos en las mismas se utilizan en modelos de aproximación a corto plazo, en general a un año, y mediano plazo, 2 a 5 años, definidos por varios autores. (Coto \& Saborio, 2006), (Robles, 2013), (D.D.N, Shyamjeet, \& Saha, 2008), (Santana, Santana, \& González, 2003). Ver Tabla 1.

\subsection{Modelado}

Los modelos de velocidad de corrosión presentan generalmente comportamientos lineales o exponenciales como función de las variables climáticas y de contaminantes atmosféricos, ya sean acumulados en el tiempo o sus promedios anuales, teniendo como variable de respuesta obtenida en los mismos es la velocidad de corrosión (Vcorr).

Dichos resultados del modelo se comparan con los valores obtenidos en campo para los distintos puntos y con el modelo de Vcorr al primer año, definido en la norma ISO 9223:2012 y con la estimación a largo plazo definida en la norma ISO 9224:1992. (ISO, 1992)

Dentro de los modelos utilizados se encuentra el definido por Feliu y Morcillo, que se define en la Ecuación 1 para el primer año (tiempo constate) (González \& Feliu, 1982) (Morcillo \& Feliu, 1993).

$V_{c o r r}=a+b \cdot T D H+c \bullet S O_{2}+d \bullet C l+e \cdot T+f \cdot H R+g \bullet P(1)$

Donde:

$V_{\text {corr }}$ es en $\mu \mathrm{m} \mathrm{x} \mathrm{año}{ }^{-1}$

$T D H$ como fracción (h de $T D H$ respecto a las h totales) $\mathrm{SO}_{2}$ y $\mathrm{Cl}^{-}$en $\mathrm{mg} \mathrm{x} \mathrm{m} \mathrm{m}^{-2} \mathrm{x} \mathrm{dia}^{-1}$

Tabla 1. Datos asociados a las estaciones de evaluación de la corrosión atmosférica

\begin{tabular}{llllll}
\hline Ubicación & \multicolumn{2}{c}{ Coordenadas } & Orientación & Altura & Observaciones \\
\hline Sabana & $9^{\circ} 56^{\prime} 33.46^{\prime \prime} \mathrm{N}$ & $84^{\circ} 6^{\prime} 11.28^{\prime \prime} \mathrm{O}$ & $\mathrm{N}$ & $1108 \mathrm{~m}$ & Edificio UEN PySA \\
Toro & $10^{\circ} 19^{\prime} 4.78^{\prime \prime} \mathrm{N}$ & $84^{\circ} 14^{\prime} 13.47^{\prime \prime} \mathrm{O}$ & $\mathrm{NO}$ & $728 \mathrm{~m}$ & C.P Toro 1 \\
Río Macho & $9^{\circ} 46^{\prime} 31.96^{\prime \prime} \mathrm{N}$ & $83^{\circ} 50^{\prime} 30.50^{\prime \prime} \mathrm{O}$ & $\mathrm{NNO}$ & $1130 \mathrm{~m}$ & P.H. Río Macho \\
Moín & $9^{\circ} 59^{\prime} 37.07^{\prime \prime} \mathrm{N}$ & $83^{\circ} 6^{\prime} 8.17^{\prime \prime} \mathrm{O}$ & $\mathrm{NNE}$ & $7 \mathrm{~m}$ & P.T. Moín \\
Diquís & $8^{\circ} 57^{\prime} 57.42^{\prime \prime} \mathrm{N}$ & $83^{\circ} 28^{\prime} 20.13 \mathrm{\prime O}$ & $\mathrm{NO}$ & $20 \mathrm{~m}$ & Palmar Norte \\
Las Pailas & $10^{\circ} 45^{\prime} 15.23^{\prime \prime} \mathrm{N}$ & $85^{\circ} 21^{\prime} 52.98^{\prime \prime} \mathrm{O}$ & $\mathrm{NO}$ & $604 \mathrm{~m}$ & C.G. Las Pailas \\
\hline
\end{tabular}

Nota: Unidad Estratégica de Negocios Proyectos y Servicios Asociados (UEN PySA), Centro de Producción (C. P.), Proyecto Hidroeléctrico (P. H.), Planta Térmica (P. T.), Centro Geotérmico (C. G.). 
$T$ es la temperatura media anual en ${ }^{\circ} \mathrm{C}$

$H R$ es la humedad relativa media anual en \%

$P$ es la precipitación anual en mm

Siendo $a, b, c, d, e, f$ y $g$ constantes del modelo matemático a calcular.

En este modelo se utilizó los datos del primer año para cada estación a fin de obtener un valor general anual para la corrosión. Se consideró también la linealidad de las variables respecto a la $V_{\text {corr }}$ y las limitaciones a la utilización de múltiples variables en función de la disponibilidad de datos.

Además estos autores también plantean una ecuación general de la $V_{\text {corr }}$ en el tiempo de la siguiente forma:

$$
\log V_{\text {corr }}=A+n \cdot \log t
$$

\section{Donde:}

$V_{\text {corr }}$ es en $\mu \mathrm{m}$ acumulado.

$t \quad$ es el tiempo en años acumulado.

$A \quad$ esta asociado con el $\log V_{c o r r}$ en el primer año.

$n \quad$ esta inversamente asociado al nivel de protección de la capa de óxido.

Para este modelo se evaluaron los valores de $A$ y $n$ para los distintos sitios.

$$
\log V_{c o r r}=a+n \bullet \log t+b \bullet C l+c \bullet S_{2}+d \bullet T D H
$$

\section{Donde:}

$$
\begin{array}{ll}
V_{\text {corr }} & \text { es en } \mathrm{g} \mathrm{x} \mathrm{m}^{-2} \\
t & \text { es el tiempo en horas acumulado }
\end{array}
$$

$T D H \quad$ como horas acumuladas
$\mathrm{SO}_{2}$ y $\mathrm{Cl}^{-}$en $\mathrm{mg} \mathrm{x} \mathrm{m}{ }^{-2}$ acumulados en el tiempo

Siendo $a, b, c$ y $d$ constantes del modelo matemático a calcular.

Para este modelo se utilizan todos los valores disponibles en cada punto, a fin de estimar las constantes de la ecuación 3 , en los distintos puntos de muestreo.

Por último se aplica a cada ubicación estudiada el modelo definido en la ISO 9223:2012, el cual considera los valores medios anuales de los parámetros climáticos para estimar un valor de la Vcorr a un año, siendo para climas tropicales la ecuación 4 (ISO, 2012) (Mikhailov, Tidblad, \& Kucera, 2004).

$$
\begin{aligned}
V_{\text {corr }}= & 1,77 \bullet \mathrm{SO}_{2} 0,52 e^{(0,020 \bullet H R-0,054 \bullet(T-10))}+\ldots \\
& 0,102 \cdot C l \\
0,62 & e^{(0,033 \bullet H R+0,040 \cdot T)}
\end{aligned}
$$

Donde:

$V_{\text {corr }}$ es en $\mu \mathrm{m} \mathrm{x} \mathrm{año-}{ }^{-1}$

$\mathrm{SO}_{2} \mathrm{y} \mathrm{Cl}^{-}$en $\mathrm{mg} \mathrm{x} \mathrm{m}{ }^{-2} \mathrm{x} \mathrm{día}^{-1}$

$\mathrm{T} \quad$ es la temperatura media anual en ${ }^{\circ} \mathrm{C}$

HR es la humedad relativa media anual en \%

Para la Ecuación 4, se definen en cada punto el valor de la ecuación según los parámetros medios anuales obtenidos en el período de análisis. Posteriormente se compara con los valores gravimétricos obtenidos en cada sitio.

El proceso de cálculo de los modelos se realiza en base a la herramienta "optimtool" de Matlab, seleccionando el sistema "Isqlin" asociado a sistemas lineales con restricciones. (Mathworks, 2013) (Díaz, Martínez-Luaces, \& Guineo-Cobs, 2003) (Miller \& Miller, 2002) (Mongay, 2005)

Para la Ecuación 2 y 3, se dispone de la restricción que el valor de $n$, tiene que ser mayor a 0 y menor a 1 . (ISO, 1992)

Además de la evaluación por los modelos antes descritos 


\subsection{Parámetros de control del modelado}

Se calculó como parámetro de control relaciones entre los valores obtenidos por los modelos y los valores obtenidos experimentales. (D.D.N, Shyamjeet, \& Saha, 2008) (Santana, Santana, \& González, 2003), (Mikhailov, Tidblad, \& Kucera, 2004) (Mathworks, 2013), (Díaz, Martínez-Luaces, \& Guineo-Cobs, 2003), (Miller \& Miller, 2002) (Mongay, 2005)

Inicialmente se calculó la suma de los Errores Residuales Cuadráticos (residual sum of squares o RSS) como evaluador del rendimiento, buscando que los valores mínimos sean menores a 1. El valor de RSS es calculado por Matlab de la siguiente forma:

$\operatorname{RSS}=\Sigma\left(E_{C}(t)-\operatorname{valor}(t)\right)^{2}$

Donde:

$E_{C}(t)$ es el valor de la función de modelado de la $V_{\text {corr }}$ a tiempo $t$

Valor $(t)$ es el valor de $V_{\text {corr }}$ a tiempo $t$

Luego se comparó dichos valores realizando una regresión lineal para determinar la recta de mejor ajuste y determinando el valor del coeficiente de correlación $\left(\mathrm{R}^{2}\right)$, asociado a la linealidad entre valores obtenidos con la ecuación utilizada y el valor experimental, cuando existe una correlación buena los valores son cercanos a 1, según la ecuación:

$R^{2}=\sqrt{\left(1 / n \cdot \Sigma(\text { fun lineal }(t)-\text { valor }(t))^{2}\right)}$

Donde:

Fun lineal $(t)$ es el valor de la función lineal de modelado de la Vcorr a tiempo $t$

Valor $(t)$ es el valor de $V_{\text {corr }}$ a tiempo $\mathrm{t}$

$n$ es el número de datos disponibles.

También se calculó el factor F de Fisher para determinar la confiabilidad del modelo frente a los datos. Se espera que el valor sea mayor a 100 .
En los casos que el tiempo no sea una variable (Ecuación 1) se controla la linealidad de las variables respecto a la $V_{\text {corr }}$, esperándose $R^{2}$ mayores a 0,7 .

Se considera además que en las iteraciones para el cálculo de las constantes de las ecuaciones de modelado, la cantidad de datos por estación debe ser mayor que el número de variables. (Miller \& Miller, 2002) (Mongay, 2005)

\section{RESULTADOS Y DISCUSIÓN}

\subsection{Obtención de datos}

La velocidad de corrosión atmosférica gravimétrica, los valores de los contaminantes atmosféricos, y los datos climáticos fueron obtenidos para cada estación y se expresan en el Tabla 2. Los datos reportados son para el período junio 2011 a julio 2013, a excepción de la estación de Las Pailas que el período considerado fue de abril de 2006 a abril de 2011.

\subsection{Análisis Ecuación (1) Modelado lineal con valores del primer año}

El análisis de la Ecuación 1 y 3 se realizó con todos los puntos, aplicando sistemas de ecuaciones lineales y cálculo a partir de Matlab, considerando todas las variables en conjunto o en grupos de 3 o más variables, considerando siempre la existencia de una constante $a$.

Se llevó a cabo un control previo de relación de linealidad entre los valores de Vcorr y las distintas variables, obteniendo que los datos de Las Pailas para el primer año no siguen la tendencia general de linealidad de las otras ubicaciones, por lo que no fueron considerados para este modelado. Esto limitó la cantidad de datos y por ende de variables a considerar (4 o menos) a partir de la Ecuación 1.

Tal como se puede observar en la Tabla 3 , se encontró que solo valores de $\mathrm{Cl}-, \mathrm{SO} 2, \mathrm{HR}$ y TDH cumplieron con el requisito de una buena linealidad con $\mathrm{V}_{\text {corr }}$, el TDH presentó un valor inferior al límite propuesto para $\mathrm{R}^{2}$. 
Tabla 2. Datos de $\mathrm{V}_{\text {corr }}, \mathrm{TDH}, \mathrm{Cl}_{\text {y SO}}$ en el tiempo para la estación de Las Pailas, Sabana, Toro, Diquís, Río Macho, Moín.

\begin{tabular}{|c|c|c|c|c|c|}
\hline $\begin{array}{l}\text { Tiempo } \\
\text { (meses) }\end{array}$ & Estación & $\begin{array}{c}\mathbf{V}_{\text {corr }}\left(g_{x} x\right. \\
\left.m^{-2}\right)\end{array}$ & TDH (h) & $\begin{array}{c}\mathrm{Cl}- \\
\left(\mathbf{m g} \times \mathbf{m}^{-2}\right)\end{array}$ & $\begin{array}{c}\mathrm{SO}_{2} \\
\left(\mathbf{m g ~} \times \mathbf{~ m}^{-2}\right)\end{array}$ \\
\hline \multirow{5}{*}{4} & Sabana & 232,9 & 2557 & 1397,35 & 355,62 \\
\hline & Toro & 258,2 & 3323 & 1275,71 & 336,01 \\
\hline & Diquís & 117,1 & 2669 & 1094,82 & 383,96 \\
\hline & Río Macho & 88,8 & 1991 & 1093,35 & 415,85 \\
\hline & Moín & 228,6 & 2832 & 1644,83 & 165,67 \\
\hline \multirow[t]{3}{*}{6} & Pailas & 145,6 & 3279 & 827,16 & 771,35 \\
\hline & Sabana & 259,6 & 4185 & 3509,22 & 1217,21 \\
\hline & Toro & 372,1 & 5670 & 3222,71 & 1521,75 \\
\hline \multirow[t]{5}{*}{8} & Diquís & 213,0 & 4600 & 2638,31 & 2086,31 \\
\hline & Río Macho & 108,8 & 3691 & 2841,92 & 1958,76 \\
\hline & Moín & 460,8 & 5598 & 3751,62 & 786,66 \\
\hline & Pailas & 288,2 & 4445 & 1501,99 & 1447,64 \\
\hline & Sabana & 353,1 & 5041 & 4460,64 & 2435,05 \\
\hline \multirow{6}{*}{12} & Toro & 470,9 & 7642 & 4359,44 & 2297,27 \\
\hline & Diquís & 263,6 & 6278 & 3414,55 & 2640,62 \\
\hline & Río Macho & 136,6 & 5153 & 3764,04 & 2892,05 \\
\hline & Moín & 564,3 & 7405 & 5201,99 & 1538,32 \\
\hline & Pailas & 384,6 & 7602 & 2144,08 & 1714,70 \\
\hline & Sabana & 457,2 & 7490 & 6370,60 & 3476,80 \\
\hline \multirow{6}{*}{18} & Toro & 564,7 & 11328 & 6255,18 & 3324,06 \\
\hline & Diquís & 307,6 & 8787 & 4632,32 & 3582,81 \\
\hline & Río Macho & 172,1 & 7421 & 5186,26 & 3983,50 \\
\hline & Moín & 769,2 & 11110 & 7620,52 & 2254,72 \\
\hline & Pailas & 433,5 & 9411 & 3199,13 & 3795,87 \\
\hline & Sabana & 512,8 & 9901 & 8714,50 & 4756,00 \\
\hline \multirow{4}{*}{24} & Toro & 679,0 & 15227 & 8528,72 & 4532,24 \\
\hline & Diquís & 378,6 & 12234 & 6513,92 & 5038,11 \\
\hline & Río Macho & 248,1 & 10688 & 7365,70 & 5657,50 \\
\hline & Moín & 1024,2 & 14804 & 10151,32 & 3003,52 \\
\hline 36 & Pailas & 531,3 & 14046 & 4848,98 & 5334,02 \\
\hline 48 & Pailas & 641,4 & 17706 & 6498,80 & 7061,71 \\
\hline 60 & Pailas & 735,3 & 22602 & 8148,62 & 8600,06 \\
\hline
\end{tabular}


Tabla 3. Ecuaciones de linealidad entre $V_{\text {corr }}$ gravimétrica y las distintas variables $\left(\mathrm{Cl}^{-}, \mathrm{SO}_{2}, \mathrm{HR}\right.$ y TDH) utilizadas en la ecuación 1

\begin{tabular}{lccr}
\hline Variable & O.O. & Pend. & $\mathbf{R}^{\mathbf{2}}$ \\
\hline $\mathbf{C l}^{-}$ & 7,886 & 0,0715 & 0,7337 \\
$\mathbf{S O}$ & 8,912 & $-0,0524$ & 0,8452 \\
$\mathbf{H R}$ & 75,598 & 0,2115 & 0,7442 \\
$\mathbf{T D H}$ & 0,471 & 0,0047 & 0,6433 \\
\hline
\end{tabular}

Nota: Pend Pendiente, O.O. ordenada en origen.

En la Tabla 3, se aprecia que la mayoría de las pendientes son positivas, indicando que existe una relación directamente proporcional, en entre las variables y la velocidad de corrosión, caso contrario para el $\mathrm{SO}_{2}$, que presenta una pendiente negativa.

Para la ecuación 1, el modelo de mejor resultado considerando en la formula general para modelado simple con TDH es:

$V_{\text {corr }}=-17,96+65,75 \cdot \mathrm{TDH}-5,34 \mathrm{SO}_{2}+4,80 \cdot \mathrm{Cl}$

$\mathrm{RSS}=143,06$

$\mathrm{F}=43,0$

$\mathrm{R}^{2}=0,935$

Mientras que considerando HR en vez de TDH, es:

$V_{\text {corr }}=-229,02+0,063 \cdot \mathrm{SO}_{2}+6,73 \cdot \mathrm{Cl}+2,345 \cdot \mathrm{HR}$

$\mathrm{RSS}=62,04$

$\mathrm{F}=103,7$

$\mathrm{R} 2=0,972$

Se determinan mejores valores de los parámetros de control, cuando se considera el promedio anual de la HR en vez de utilizar TDH, dado que el valor de RSS son menores, lo cual sugiere que el modelo utilizando HR presenta menor error que el de TDH, mientras que los de $\mathrm{F}$ y $\mathrm{R}^{2}$ son mayores, indicando que existe una mejor correlación entre los datos utilizando el promedio de HR anual.

Los valores obtenidos en estas ecuaciones para RSS son elevados, mientras que aunque $\mathrm{R}^{2}$ es relativamente cercano a 1 , los valores de $\mathrm{F}$ no expresan una situación adecuada de la relación entre los datos y la función de estimación.

La curva de estimación (Ecuación 8) se compara con los valores reales de $\mathrm{V}_{\text {corr }}$, tal como se muestra en la Figura 1, observándose una ligera subvaloración de la corrosión real cuando la $\mathrm{V}_{\text {corr }}$ está entre $30 \mu \mathrm{m}$ y $60 \mu \mathrm{m}$, mientras que sobrevalora fuera de ese rango.

\subsection{Análisis Ecuación (2) Modelado Logarítmico}

En el caso de la Ecuación 2, el modelado se realiza para cada punto considerando, expresan las constantes en cada sitio en la Tabla 4.

En general esta ecuación presenta buena correlación de linealidad para todos los sitios, debido a que los valores de $\mathrm{R}^{2}$, se encuentran entre 0,9409 y 0,9978 .

Los valores de la constante A, en todas las estaciones son similares a $\operatorname{los}$ de $\log \mathrm{V}_{\text {corr }}$ del primer año.

La constante $n$ obtenida en la Tabla 4, presenta valores entre 0,5 y 0,9 , asociado según la bibliografía a procesos de estabilización de los óxidos superficiales con el tiempo. En general para lugares alejados de la costa los valores de $\mathrm{n}$ se concentrarían entre 0,5 y 0,7 , valores considerados como parcialmente protectores. (Morcillo, Almeida, Rosales, Uruchurtu, \& Morrocos, 1998), (Robles, 2013), (Corvo et al, 2008), (Chico, Otercr, Mariaca, \& Morcillo, 1998), (Meira, Andrade, Alonso, Padaratz, \& Borba, 2008)

En base al caso más extenso de Las Pailas, esto puede asociarse a que el proceso de estabilización de los óxidos en la superficie es lento, del orden de 2 a 3 años, para obtener una velocidad de corrosión estable, como se puede apreciar en la Tabla 6.

\subsection{Análisis Ecuación (3) Modelado Exponencial}

Para la aplicación de la Ecuación 3 debe considerarse la cantidad de datos disponibles para cada sitio en relación a la cantidad de variables 


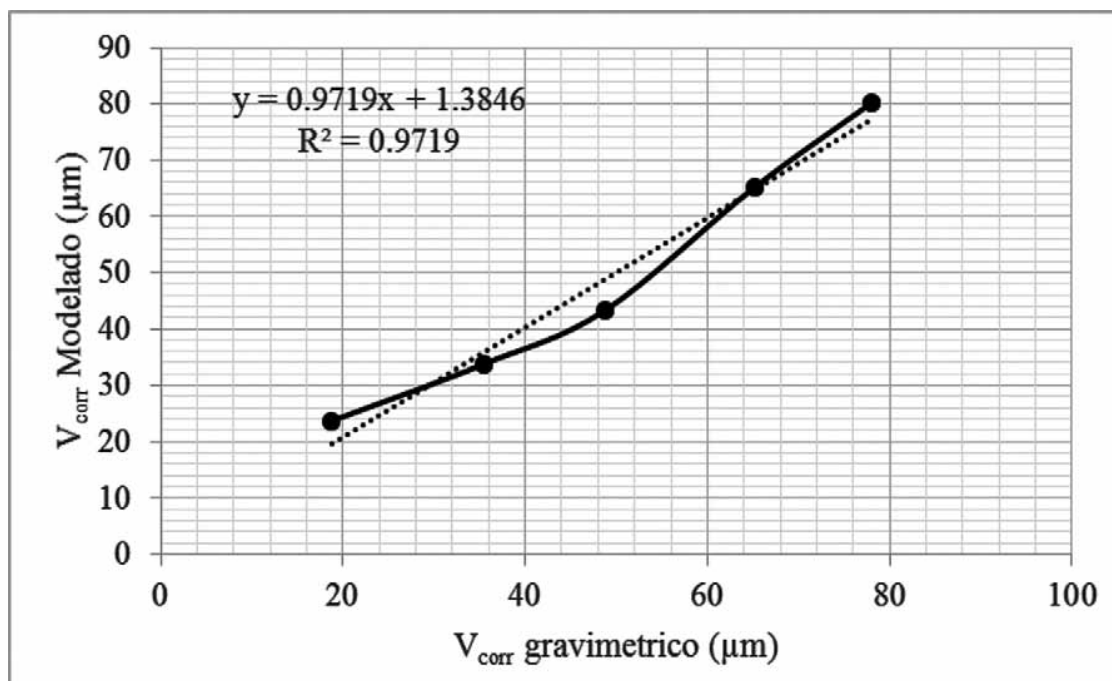

Figura 1. Relación entre la $\mathrm{V}_{\text {corr }}$ Modelado $(\mu \mathrm{m})$ en el primer año calculada por la Ecuación 8 , respecto a los valores de $\mathrm{V}_{\text {corr }}$ gravimétrico $(\mu \mathrm{m})$ utilizados para el modelado.

Tabla 4. Valores de las constantes para la Ecuación 2 en cada sitio.

\begin{tabular}{lcccc}
\hline Sitio & $\mathbf{A}$ & $\mathbf{n}$ & $\mathbf{R}^{\mathbf{2}}$ & $\log \mathbf{V}_{\text {corr }}(\mathbf{a n ̃ o ~ 1 )}$ \\
\hline Las Pailas & 1.2224 & 0,6905 & 0,9793 & 1,2632 \\
Sabana & 1.6425 & 0.5942 & 0.9428 & 1.6470 \\
Toro & 1.7504 & 0.6758 & 0.9955 & 1.7733 \\
Río Macho & 1.2664 & 0.6198 & 0.9409 & 1.2323 \\
Moín & 1.8478 & 0.9107 & 0.9978 & 1.8518 \\
Diquís & 1.4894 & 0.7303 & 0.9891 & 1.5085 \\
\hline
\end{tabular}

Tabla 5. Valores de las constantes para la Ecuación 3 en cada sitio, para el sistema con menor RSS.

\begin{tabular}{|c|c|c|c|c|c|c|c|c|}
\hline Sitio & $\mathbf{a}$ & $\mathbf{n}$ & b $\mathrm{Cl}^{-}$ & $\mathrm{c} \mathrm{SO}_{2}$ & d TDH & RSS & $\mathbf{F}$ & $\mathbf{R}^{2}$ \\
\hline Las Pailas & $-1,275$ & 0,961 & $1,29 \times 10-4$ & $-7,76 \times 10-5$ & $-3,18 \times 10-5$ & $8,23 \times 10^{-4}$ & 2069 & 0,9976 \\
\hline Sabana & 1,879 & 0,143 & $-5,52 \times 10-5$ & $1,50 \times 10-4$ & ND & $8,96 \times 10^{-4}$ & 295 & 0,9900 \\
\hline Toro & 1,149 & 0,375 & $-1,19 \times 10-4$ & $2,45 \times 10-4$ & ND & $2,03 \times 10^{-5}$ & 15778 & 0,9998 \\
\hline Río Macho & 1,110 & 0,215 & $-5,58 \times 10-5$ & ND & $7,30 \times 10-5$ & $1,48 \times 10^{-4}$ & 2442 & 0,9988 \\
\hline Moín & $-1,180$ & 1,000 & $1,85 \times 10-5$ & $-7,91 \times 10-5$ & ND & $3,04 \times 10^{-4}$ & 2458 & 0,9988 \\
\hline Diquís & $-0,683$ & 0,797 & $9,42 \times 10-5$ & ND & $-6,00 \times 10-5$ & $4,83 \times 10^{-6}$ & 94612 & 0,9999 \\
\hline
\end{tabular}

Nota: No disponible (ND) 
Tabla 6. Valores de $\mathrm{V}_{\text {corr }}$ según la ISO 9223:2012, para lo sitios considerados y la evolución de $\mathrm{V}_{\text {corr }}$ en los mismos con el tiempo, expresado en $\mu \mathrm{m} \mathrm{x} \mathrm{año-1.}$

\begin{tabular}{|c|c|c|c|c|c|c|}
\hline Sitio & $\mathbf{V}_{\text {corr }}$ & $V_{\text {corr }}$ año1 & $V_{\text {corr }}$ año 2 & $V_{\text {corr }}$ año 3 & $V_{\text {corr }}$ año 4 & $V_{\text {corr }}$ año 5 \\
\hline Las Pailas & 19,92 & 40,31 & 30,32 & 24,79 & 22,46 & 20,60 \\
\hline Sabana & 20,70 & 44,00 & 35,72 & ND & ND & ND \\
\hline Toro & 28,46 & 64,26 & 47,35 & ND & ND & ND \\
\hline Río Macho & 22,33 & 18,50 & 17,16 & ND & ND & ND \\
\hline Moín & 25,40 & 77,00 & 71,63 & ND & ND & ND \\
\hline Diquís & 20,35 & 34,93 & 26,30 & ND & ND & ND \\
\hline
\end{tabular}

Nota: No Disponible (ND)

a poder considerar a fin de que el resultado no sea único y se presente algún nivel de modelado con la ecuación. En este sentido Las Pailas tiene mayor número de datos lo que permite considerar todas las variables o combinaciones de 2 a 4 , a fin de obtener las constantes de la ecuación. Por otro lado los restantes puntos tienen menor número de datos lo que restringe la ecuación de modelado a la utilización de 2 o 3 variables, con las correspondientes limitaciones en la cantidad de constantes para la ecuación de modelado.

Por este motivo para estos sitios se consideró determinar cuál sería la combinación de mejor aproximación, en procesos dependientes del tiempo (con las constantes a y n) y con una o dos variables adicionales de las posibles $\left(\mathrm{Cl}^{-}, \mathrm{SO}_{2}\right.$ y TDH), además la condición de que $0<\mathrm{n} \leq 1$. Los valores expresados en la Tabla 5 son los de mejor RSS obtenidos en cada sitio.

Las constantes $a$ y $n$ son diferentes a las obtenidas en la Ecuación 2, debido a que el modelado se torna más complejo y restrictivo al aumentar la cantidad de variables.

Como variables principal aparece el $\mathrm{Cl}^{-}$, asociado generalmente al $\mathrm{SO}_{2}$ y en algunos casos al TDH, cuando los valores de $\mathrm{SO}_{2}$ son más altos.

Las constantes asociadas al Cl- están en el orden de (1 a 13) x 10-5, mientras que su signo es variable pero opuesto a los signos de las otras variables consideradas ( $\mathrm{SO}^{2} \mathrm{y} / \mathrm{o} \mathrm{TDH}$ ).
En cuanto a la constante del $\mathrm{SO}_{2}$, la misma está en el orden de (7 a 25) x10-5 y la de TDH entre (3 a 8$) \cdot 10^{-5}$.

Los valores de RSS, F y R² plantean buenas correlaciones entre el modelo en cada sitio y los datos reales.

La relación entre ecuaciones de distintos sitios muestran linealidad con $\mathrm{R}^{2}$ mayor a 0,6 , pero con pendientes mucho mayores a 1 y ordenadas en el origen distintas de cero. Esto implica que cada función solo tendría aplicación local. La figura 2 muestra una de las mejores relaciones, al aplicar la ecuación de Sabana con los datos de Toro, para relacionar los valores de $\mathrm{V}_{\text {corr }}$ modelado con los de $\mathrm{V}_{\text {corr }}$ obtenido en el sitio.

\subsection{Análisis Ecuación (4) Modelado según ISO 9223:2012 y resultados de velocidad de corrosión atmosférica gravimétricos}

En cuanto a la Ecuación 4, definida en la ISO 9223:2012, bajo las condiciones de clima tropical se aplican para todos los sitios, evaluándose el resultado respecto a los valores del primer año y a los demás periodos disponibles.

La Tabla 6 muestra la relación entre las velocidades de corrosión obtenidas por la ecuación 4 y los valores gravimétricos experimentales obtenidos en el tiempo para cada sitio, en general 


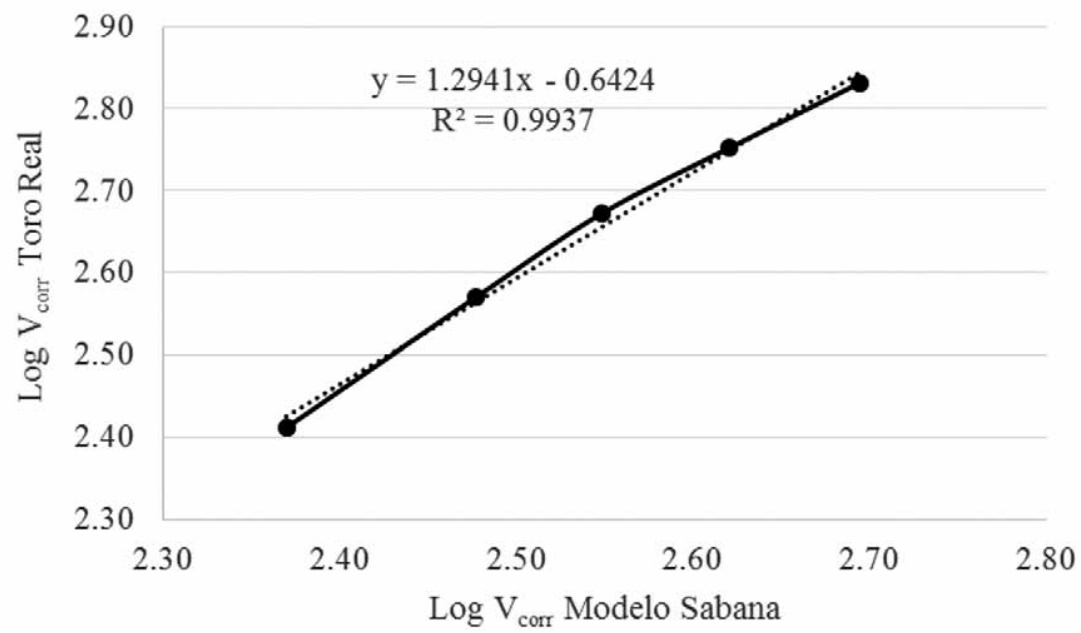

Figura 2. Relación entre $\log \mathrm{V}_{\text {corr }}\left(\mathrm{g} \mathrm{x} \mathrm{m}^{-2}\right)$ obtenida con el modelo de Sabana, aplicando en ella los datos de Toro y su relación con los valores reales de $\log \mathrm{V}_{\text {corr }}\left(\mathrm{g} \mathrm{x} \mathrm{m}^{-2}\right)$ obtenidos en Toro.

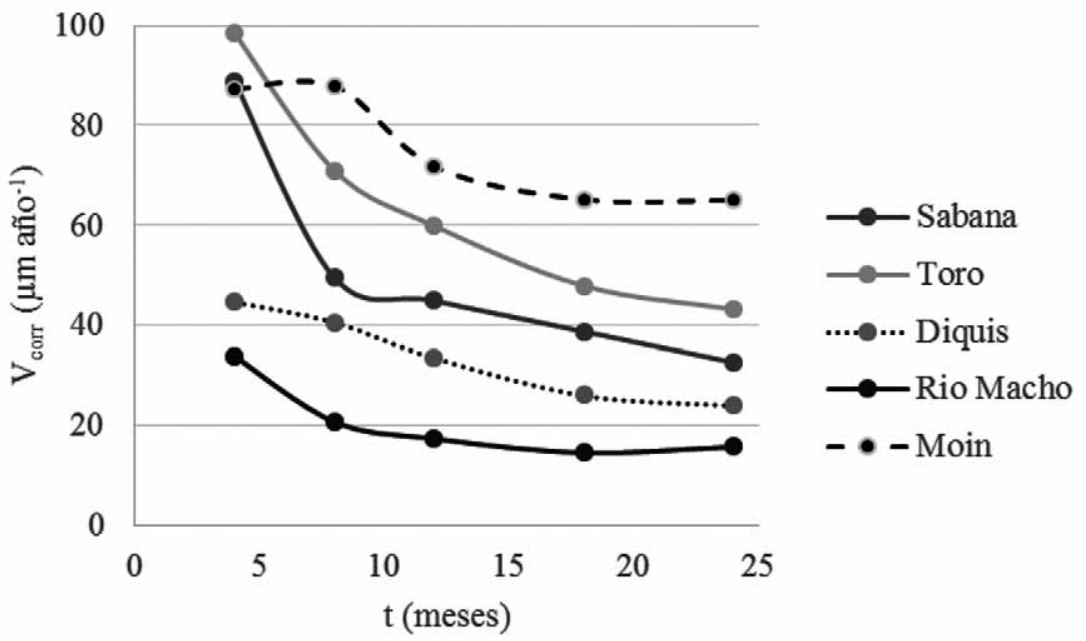

Figura 3. Evolución de $\mathrm{V}_{\text {corr }}(\mu \mathrm{m}$ año-1) con el tiempo para los 5 sitios analizados durante dos años. 
el valor de $\mathrm{V}_{\text {corr }}$ aportado por la Ecuación 4, resulta en la mayoría de los casos, menor al valor para el primer año, ajustándose con el tiempo a un valor más cercano.

La tendencia general vista en Las Pailas es que los valores de corrosión decrecen los 2 a 3 primeros años hasta estabilizarse en un valor.

Delos sitios considerados solo las velocidades de corrosión atmosférica obtenida gravimétricamente para Río Macho presenta valores por debajo de lo estimado por el modelo, asociado posiblemente a un bajo valor de $\mathrm{HR}$ promedio y un mayor nivel de $\mathrm{SO}_{2}$ que podría estar afectando la $\mathrm{V}_{\text {corr. }}$

\subsection{Estimación de $\mathrm{V}_{\text {corr }}$ según la ISO 9224:1992}

La evaluación a largo plazo de la norma ISO 9224:1992 (ISO, 1992) considera una ecuación similar a la Ecuación 2 para el modelado, plantean valores de la constante $n$ (B2 en la norma) del orden de 0,575 (valor dentro del rango obtenido para la constante $n$ de la Ecuación 2).
La misma muestra los valores máximos definidos por la norma para cada categoría de corrosión atmosférica, en función del tiempo, lo cual se expresa en la Tabla 7.

Los valores máximos esperados por la norma ISO 9224 no son superados por lo encontrado gravimétricamente en cada sitio. Mientras que los valores modelados, considerando el uso de la $\mathrm{V}_{\text {corr }}$ del primer año, para estimar la $\mathrm{V}_{\text {corr }}$ los siguientes años resultan bastante concordantes a excepción de Río Macho y Moín. Ambos presentan valores modelados menores que los obtenidos.

En caso de Moín quizás asociado a que el valor de la constante $\mathrm{n}$ es muy superior a 0,575 , siendo la corrosión (en $\mu \mathrm{m}$ año-1) casi constante después del primer año. Algo similar ocurre en Río Macho, que, aunque con un valor de n más similar a B2, en el segundo año parece haberse estabilizado la $\mathrm{V}_{\text {corr }}$, acercándose más al límite superior de la corrosión esperada para una categoría C2.

La evolución de la $\mathrm{V}_{\text {corr }}\left(\mu \mathrm{m}\right.$ año $\left.{ }^{-1}\right)$ puede apreciarse en la Figura 3, para los 5 sitios analizados en simultaneo durante dos años.

Tabla 7. Valores de $\mathrm{V}_{\text {corr }}$ según la ISO 9223:2012, para lo sitios considerados y la evolución de $\mathrm{V}_{\text {corr }}$ en los mismos con el tiempo, expresado en $\mu \mathrm{m} x$ año-1.

\begin{tabular}{|c|c|c|c|c|c|c|c|}
\hline Sitio & $\begin{array}{c}\text { Categoría ISO } \\
\text { 9223:1992 }\end{array}$ & $\mathbf{V}_{\text {corr }}$ año1 & $\begin{array}{c}V_{\text {corr MAX }} \\
\text { año1 }\end{array}$ & $\mathbf{V}_{\text {corr }}$ año2 & $\begin{array}{c}V_{\text {corr MAX }} \\
\text { año2 }\end{array}$ & $V_{\text {corr }}$ año5 & $\begin{array}{c}\mathbf{V}_{\text {corr MAX }} \\
\text { año5 }\end{array}$ \\
\hline Las Pailas & $\mathrm{C} 3$ & 40,31 & 50 & 60,64 & 72 & ND & ND \\
\hline Sabana & $\mathrm{C} 3$ & 44,00 & 50 & 71,44 & 72 & ND & ND \\
\hline Toro & $\mathrm{C} 4$ & 64,26 & 80 & 94,70 & 115 & ND & ND \\
\hline Río Macho & $\mathrm{C} 2$ & 18,50 & 25 & 34,32 & 36 & ND & ND \\
\hline Moín & $\mathrm{C} 4$ & 77,00 & 80 & 143,26 & 115 & ND & ND \\
\hline Diquís & $\mathrm{C} 3$ & 34,93 & 50 & 52,60 & 72 & ND & ND \\
\hline
\end{tabular}

Nota: No Disponible (ND) 


\section{CONCLUSIONES}

- Al desarrollar la Ecuación 1, asociada a valores de $\mathrm{V}_{\text {corr }}$ del primer año, la misma presenta limitaciones importantes, teniendo altos valores de RSS y bajos de F. Se considera que esta ecuación dará un valor meramente cualitativo. La misma posee una mejor correlación con la HR que con el TDH.

- La Ecuación 2, desarrollada para cada sitio muestra buenas correlaciones de $\mathrm{R}^{2}$, pero los valores de $\log \mathrm{V}_{\text {corr }}$ en el primer año no tienen relación con el valor obtenido para la constante A. Paralelamente los valores obtenidos de $\mathrm{n}$ se encuentran en un rango de 0,5 a 0,9, los cuales se asocian a productos de corrosión poco protectores.

- El desarrollo de la Ecuación 3, presenta un modelo de mejor ajuste para cada uno de los sitios, obteniéndose buenos valores de los parámetros de control (RSS, F y R²), pero no así de la aplicación de las mismas en otros sitios. Las variables principales sobre Vcorr son el Cl- y $\mathrm{SO}_{2}$, y TDH, dependiendo del sitio.

- La ecuación planteada por la norma ISO 9223:2012, Ecuación 4, presenta un ajuste pobre a valores asociados a la $\mathrm{V}_{\text {corr }}$ en el primer año, para las estaciones utilizadas, pero parece ser un valor a adecuarse a largo plazo.

\section{AGRADECIMIENTOS}

Se agradece de forma especial el apoyo brindado por parte del Centro de Desarrollo Tecnológico del Instituto Costarricense de Electricidad, para el desarrollo de los modelados en este trabajo.

\section{REFERENCIAS}

ASTM. (1976). ASTM G50-76 (Reapproved 2003) Standard Practice for Conducting Atmospheric Corrosion Tests on Metals.

Chico, B., Otercr, E., Mariaca, L., \& Morcillo, M. (1998). La corrosión en atmósferas marinas. Efecto de la distancia a la costa. Rev. Met. 34 (mayo), 71-74
Corvo, F., Perez, T., Dzib, L. M., Castaneda, A., Gonzalez, E., \& Perez, E. (2008). Outdoor-indoor corrosion of metals in tropical coastal atmospheres. Corrosion Science, 220-230.

Corvo, F., Perez, T., Dzib, L., Martin, Y., Castaneda, A., \& Gonzalez-Sanchez, J. (2008). Considerations on the estimation of TOW according to ISO 9223 standard. Corrosion Science, 206-219.

Coto, C., \& Saborio, L. (2006). Corrosión atmosférica en plantas del Instituto Costarricense de Electricidad. LATINCORR.

D.D.N, S., Shyamjeet, J., \& Saha, K. (2008). Role of climatic conditions on corrosion characteristics . Corrosion Science , 93-110.

Davies, J. (1998). Metals Handbook. Ohio: ASM International.

Díaz, V., Martínez-Luaces, V., \& Guineo-Cobs, G. (2003). Corrosión atmosférica: validación de modelos empleando técnicas estadísticas. Revista Metal, Madrid, 244-248.

González, J. A. \& Feliu, S. (1982). Corrosión atmosférica del Al, Cu, Fe y Zn: (III Parte). Posibilidades de las técnicas electroquímicas para estimar la velocidad de corrosión instantánea en atmósferas controladas", Madrid: CENIM.

ISO. (1992). ISO 9223 Corrosion of metals and alloys Corrosivity of atmospheres Classification.

ISO. (1992). ISO 9224 Corrosion of metals and alloys Corrosivity of atmospheres guiding values for the corrosion categories.

ISO. (1992). ISO 9225 Corrosion of metals and alloys corrosivity of atmosphere measurement of pollution.

ISO. (1992). ISO 9226 Corrosion of metals and alloys Corrosivity of atmosphere Determination of corrosion rate of satndard specimens for evluation of corrosivity.

ISO. (2012). ISO 9223 Corrosion of metals and alloys Corrosivity of atmospheres, classification.

Mathworks. (2013). Matlab. Obtenido de www. mathworks.com/products/matlab/

Meira, G., Andrade, C., Alonso, C., Padaratz, I., \& Borba, J. (2008). Modelling sea salta transporte and deposition in marine zone 
atmosphere zopne, a tool for corrosion stiudies. Corrosion Science, 2724-2731.

Mikhailov, A., Tidblad, J., \& Kucera, V. (2004). The Classification System of ISO 9223 Standard and the Dose-Response Functions Assessing the Corrosivity of Outdoor Atmospheres. Protection of metals, 541-550.

Miller, M., \& Miller, M. (2002). Estadística Quimiometría para química analítica. Madrid: Pearson.

Mongay, C. (2005). Quimiometria. Valencia: Edicuacio Materials.

Morcillo, M., \& Feliu, S. (1993). Mapas de España de Corrosividad. Madrid: CYTED.

Morcillo, M., Almeida, E., Rosales, B., Uruchurtu, J., \& Morrocos, M. (1998). Corrosión y Protección de metales en las atmosferas iberoamerica, Parte 1: mapas iberoamericanos de corrosividad atmosferica. Madrid: Programa CYTED.

Robles, J. (2013). Evaluación de la corrosion atmosferica en tres zonas geograficas de Costa Rica por medio de técnicas electroquimicas y gravimetricas. (Tesis de Licenciatura en Química Industrial). Universidad Nacional, Heredia.
Santana, J., Santana, F., \& González. (2003). The effect of environmental and meteorological variables on atmospheric corrosion of carbon steel, copper, zinc and aluminium in a limited geographic zone with different types of environment. Corrosion Science, 799-815.

Solano, J., \& Villalobos, R. (2000). Regiones y subregiones climáticas de Costa Rica. San José: Instituto Meteorológico Nacional.

\section{SOBRE LOS AUTORES:}

Luis Garita Arce. Investigador en Centro de Investigación en Corrosión, Instituto Costarricense de Electricidad.

Correo electrónico: garitaluis@gmail.com

Javier Rodriguez Yañez. Investigador en Centro de Investigación en Corrosión, Instituto Costarricense de Electricidad.

Correo electrónico: urutico@gmail.com

Javier Robles Cordero. Investigador del Centro de Investigación en Corrosión, Instituto Costarricense de Electricidad.

Correo electrónico: jroblesco@ice.go.cr 